\title{
Artificial Neural Network Based Lung Cancer Detection for PET/CT Images
}

\author{
Punithavathy Kannuswami ${ }^{1 *}$, Sumathi Poobal ${ }^{2}$ and M. M. Ramya ${ }^{3}$ \\ 'Department of Electronics and Communication Engineering, Hindustan Institute of Technology \& Science, \\ Padur, Chennai -603103, Tamil Nadu, India; punithavm@hindustanuniv.ac.in \\ 2Department of Electronics and Communication Engineering, KCG College of Technology, Karapakkam, \\ Chennai - 600097, Tamil Nadu, India; sumathipoobal@gmail.com \\ ${ }^{3}$ Centre for Automation and Robotics, Hindustan Institute of Technology \& Science, \\ Padur, Chennai - 603103, Tamil Nadu, India; mmramya@hindustanuniv.ac.in
}

\begin{abstract}
Background/Objectives: To develop an Artificial Neural Networks (ANN) based Computer Aided Diagnosis system (CAD) using texture and fractal features to detect lung cancer from Positron Emission Tomography/Computed Tomography (PET/ CT) images. Methods/Statistical Analysis: Methods such as Wiener filtering and fuzzy image processing were used to suppress noise and improve the contrast respectively in lung PET/CT images. Texture and fractal features were analyzed to extract significant features. ANN with optimal network parameters was designed to train and test the dataset. Total of 1072 training samples and 80 testing samples were used to evaluate the performance of the system. Findings: The proposed fuzzy enhancement played a vital role in improving the detection of lung cancer. 13 significant features were identified ( 3 texture features and 10 fractal features) for detection of cancerous regions. Proposed method of CAD system yielded better classification accuracy for training and testing with Levenberg-Marquardt back propagation, learning rate $=0.3$, momentum $=0.9$ and 20 hidden neurons. The training accuracy produced by texture, fractal and combined features were $92.4 \%, 98.1 \%$ and $98.5 \%$ respectively. The testing accuracy achieved with the proposed method for texture, fractal and combined features were $91.3 \%, 95 \%$ and $92.5 \%$. Proposed classifier with fractal features yielded a better testing accuracy than texture and combined features. Improvements/Applications: Deep learning algorithms may be implemented to improve the accuracy of the detection. Developed CAD system can act as a decision support system to assist radiologists in lung cancer diagnosis.
\end{abstract}

Keywords: Artificial Neural Networks, Computer Aided Diagnosis, Fuzzy Enhancement, Lung Cancer, Texture and Fractal Features

\section{Introduction}

Cancer is a life threatening disease nowadays. Lung cancer takes the first position in men and second position in women among various cancers. There has been a steady increase in lung cancer occurrence in the last 10 years ${ }^{1}$. Lung cancer is the leading contributor in cancer-related deaths, accounting for 1.38 million cancer deaths per year worldwide ${ }^{2}$. The 5 year survival is only $14 \%$ and it has not been improved in last few decades $\underline{\underline{3}}$. In Indian subcontinent, lung cancer is most often interpreted as tuberculosis and hence diagnosed very lately in the terminal stages ${ }^{4}$. Timely diagnosis and better treatment options can improve the patient's survival time. Image processing techniques play a phenomenal role in lung cancer detection with a potential of diagnosing the disease in its early stages and distinguishing tuberculosis from lung cancer ${ }^{5}$. PET/CT is currently used in lung cancer diagnosis, staging, treatment planning and monitoring with the combined functional and anatomical information acquired at the same time ${ }^{6}$. Most challenging factor with

*Author for correspondence 
PET/CT is the miss-diagnosis due to the overlapping of the value in diagnostic feature, Standardized Uptake Value (SUV) ${ }^{z}$.

CAD systems play a vital role in medical image analysis. CAD systems make use of radiological image processing and artificial intelligence to identify suspicious image features and use a classification algorithm like linear discriminate analysis, ANN or Support Vector

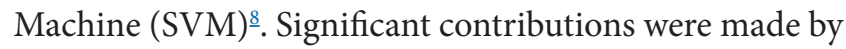
many researchers by developing a CAD system in various cancer diagnoses including lung cancer diagnosis using chest radiographic images and lung CT images. CAD systems implemented for lung cancer detection by several researchers have been critically reviewed and noted that promising results were achieved by several systems, but still the level of automation can be improved in future?

ANN has been implemented to develop intelligent systems and successfully applied to broader spectrum of areas like financial, data mining, medical, industrial, robotics \& control, aeronautics etc. Multilayer Perception (MLP) was developed to diagnose the disease present in the liver from liver ultrasound images. Mixed features and GLRLM features produced testing accuracy of $92.5 \%$ and $95 \%$ respectively ${ }^{10}$. Neural network was applied to diagnose the thyroid tumor and tried with thirteen learning algorithms. Better classification results were obtained with Polak-Ribiere conjugate gradient algorithm for 5 hidden neurons ${ }^{11}$. SVM classifiers were constructed to detect the cervical cancer from Magnetic Resonance Images (MRI) using texture and transform features. Results showed that the texture features outperformed transform features ${ }^{2}$. Feed-forward Back Propagation Neural Network (BPNN) produced better performance with Levenberg-Marquardt back propagation and scaled conjugate gradient back propagation in classifying brain tumor images $\frac{13}{}$.

BPNN classifier with 30 hidden neurons and a learning rate of 0.3 was tested on a dataset of 10 chest X-ray images and obtained $80 \%$ accuracy for lung cancer classification ${ }^{14}$. First order texture features were used in training and testing an ANN system to detect lung cancer from CT images ${ }^{15,16}$. CAD system was developed for early detection of lung cancer using region based segmentation. Extracted features such as color and first order statistical texture features were fed to the ANN for classification. The system is semi-automatic which depends upon the manual selection of the initial seed point for region based segmentation $\underline{17}$.
Few attempts have been made to implement a complete automated system in cancer diagnosis. Second order statistical texture features were extracted from 250 lung CT images and significant features were selected by using ant colony optimization technique. Two machine learning systems such as SVM and ANN have been tried to classify the lung CT images. ANN resulted in a better accuracy of $98.4 \%$ compared to $93.2 \%$ by SVM ${ }^{18}$. Hop field neural network based classification was implemented to detect lung cancer and observed that an accuracy of $98 \%$ was achieved $\underline{19}$. CAD system for an automatic lung nodule detection from radiographic images was investigated using a two level ANN. 96\% sensitivity was achieved for radiographic images using curvature peaks ${ }^{20}$. Fractal analysis has been applied as a powerful tool in biomedical engineering to characterize the complex morphology and suitable for distinguishing between pathological images. Use of fractal features in lung cancer diagnosis can improve the diagnostic accuracy 21 .

This study focuses on establishing a CAD system using BPNN to analyze the performance of the classifier with the extracted texture, fractal features from lung PET/ CT images for lung cancer diagnosis.

\section{Materials and Methods}

Lung PET/CT images, collected from Anderson diagnostics \& Labs, Chennai, captured from 46 men and 36 women in the age group of $28-76$ years were utilized for this study. The dataset included 48 abnormal and 34 normal studies. Abnormal images comprised of non-small cell lung cancer of 12, 21 and 15 images in stage II, III and IV respectively. 1152 samples were taken from these 82 images considering the background, cancer regions and boundary between the normal and cancer regions. Texture and fractal features were extracted and analyzed to determine the classification rule.

The flow diagram describing the sequence of operations of the proposed method is shown in Figure 1. Lung $\mathrm{PET} / \mathrm{CT}$ images were pre-processed to de-noise and enhance the contrast of the image. Texture and fractal features were extracted from the pre-processed images. Significant features were identified and used to train and test the classifier. 


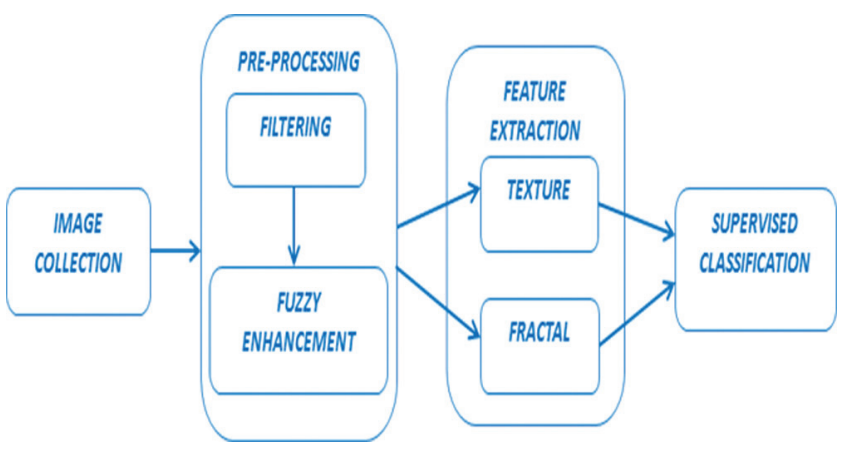

Figure 1. Flow diagram of the proposed lung cancer detection and classification system.

\subsection{Image Pre-Processing}

Quality of the acquired PET/CT images was reduced by the variations in the contrast and additive noise present ${ }^{22}$. These degradations in the image affect the accuracy of cancer diagnosis. Wiener filtering has been well explored for its de-noising performance. Wiener filtering was applied on lung CT images to remove the additive noise; with edges and fine details of lungs preserved $\underline{23}$. Hence Wiener filtering was used in this study to de-noise the additive noise present in lung PET/CT images. Adaptive way of wiener filtering produced optimal results when compared to other linear filtering techniques.

Lung PET/CT images have different characteristics due to different densities of cancer cells, variation in size and shape of cancer affected regions. This makes the boundaries and regions of lung PET/CT images fuzzy in nature. Traditional image enhancement methods do not improve the quality of uncertain/vague medical images. Hence, fuzzy image processing was used to handle the imprecise, uncertain and vague information in lung PET/ CT images. Cubic function was used as membership function to provide much soft thresholding and unique defuzzification based on a threshold ' $\mathrm{T}$ ' of the membership assigned image $\mu^{\prime}(i, j)$ and a gain factor ' $\mathrm{K}$ ' to vary the contrast of Region of Interests (ROIs) and non-ROIs. Fuzzy enhanced image is obtained by using:

$$
I_{1}(i, j)=\left\{\begin{array}{cl}
0 & \text { if } \mu^{\prime}(i, j) \leq 0 \\
255 * K * \mu^{\prime}(i, j) & \text { if } 0<\mu^{\prime}(i, j)<T \\
255 & \text { if } \mu^{\prime}(i, j) \geq T
\end{array}\right.
$$

\subsection{Texture Features}

Texture is an important spatial property of an image which provides useful information about the spatial arrange- ment of the pixels and their relationship to surrounding. Second order statistical texture features extracted from gray level co-occurrence (GLCM) matrix were useful in image classification ${ }^{24}$. Texture analysis suits well for lung cancer detection because texture features provide unique information about the spatial variation of the pixels in a region. An earlier study on texture features was performed by the authors for detection of lung cancer. Out of 14 features extracted, the study identified 3 significant texture features $\underline{25}$. Table 1 shows the significant second order statistical texture features used in the proposed study.

Table 1. Significant texture features

\begin{tabular}{|c|c|c|c|}
\hline S. No & $\begin{array}{l}\text { Feature } \\
\text { number }\end{array}$ & $\begin{array}{l}\text { Texture } \\
\text { feature }\end{array}$ & Formula \\
\hline 1 & $t f_{1}$ & $\begin{array}{l}\text { Auto- } \\
\text { correlation }\end{array}$ & $t f_{1}=\sum_{i} \sum_{j}(i j) p(i, j)$ \\
\hline 2 & $t f_{8}$ & $\begin{array}{l}\text { Sum } \\
\text { average }\end{array}$ & $t f_{8}=\sum_{i=2}^{2 N_{g}} i p_{x+y}(i)$ \\
\hline 3 & $t f_{10}$ & $\begin{array}{l}\text { Sum } \\
\text { variance }\end{array}$ & $\begin{array}{c}t f_{10}=\sum_{i=2}^{2 N_{g}}\left(i-t f_{9}\right)^{2} i p_{x+y}(i) \\
\text { where } t f_{9}-\text { sum entropy }\end{array}$ \\
\hline
\end{tabular}

\subsection{Fractal Features}

Fractal analysis has been used in medical images to characterize the complex, irregular structures which cannot be characterized by traditional topological dimension. Fractal analysis has been found useful in lung cancer detection as the lung and branching vessels exhibit fractal nature. Fractal Dimension (FD) is a useful parameter in characterizing random structures. Differential box counting (DBC) was used to compute the FD of gray scale images and shown in Figure 2.

In DBC method, an image of size $N \times N$ was considered as a 3 -Dimensional space with $(x, y)$ representing the pixel position and the third axis $(z)$ denote the pixel intensity value. The $(x, y)$ space was covered with grids of size, $\mathrm{s}$ $\times \mathrm{s}$, where $N / 2 \geq s \geq 2$. Each grid in the $(x, y)$ space had a column of grid with size $s \times s \times h$ in the third axis, where $h$ is the height of the grid. The process was carried out on a moving window of size $5 \times 5$ and repeated for various scales $(r=1 / s)$. Number of boxes required to cover the grid $(i, j)$ was calculated using Equation (2) where $l$ and $k$ 


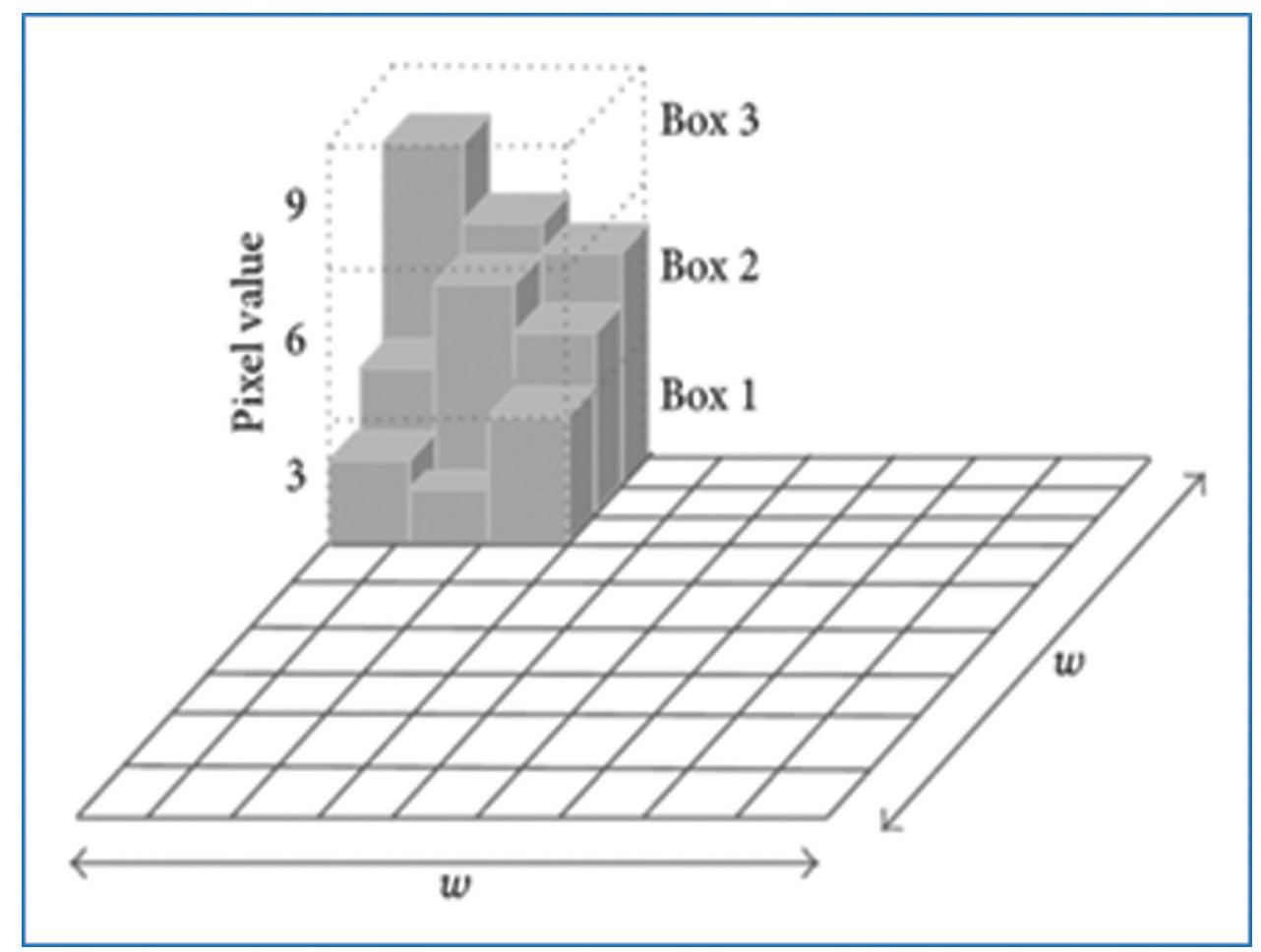

Figure 2. Differential box counting method of computing FD.

are the boxes containing maximum and minimum intensity values respectively.

$$
n_{r}(i, j)=l-k+1
$$

The total number of grids which contain image information is given by:

$$
N(r)=\sum_{i, j} n_{r}(i, j)
$$

$F D$ was computed by using the formula:

$$
F D=\log (N(r)) / \log (1 / r)
$$

Lacunarity, complementary information of fractal dimension was used to measure the amount of in-homogeneity in an image by analyzing the distribution of gaps in an object and defined as:

$$
\text { Lacunarity }=\left(\text { Variance } \mathrm{Mean}^{2}\right)
$$

Images with different textures may result in a same $F D$ as irregularity measurement is better, but spatial arrangements of the elements are not well considered. 6 fractal features $f_{1}-f_{6}$ were identified and derived to solve this issue in segmentation of various natural and medical images $\frac{26,27}{}$. These fractal features were derived from the fractal transformed images of fuzzy enhanced image. For each fractal feature $f f_{1}-f f_{6}, F D_{\text {avg }}$, and lacunarity were found and hence 12 fractal features were extracted in lung cancer detection from PET/CT images.

The images from the fractal features were extracted as given below:

$$
\begin{gathered}
I_{2}(i, j)=\left\{\begin{array}{cc}
I_{1}(i, j)-L_{1} ; & \text { if } I_{1}(i, j)>L_{1} \\
0 & \text { otherwise }
\end{array}\right. \\
I_{3}(i, j)=\left\{\begin{array}{c}
255-L_{2} ; \quad \text { if } I_{1}(i, j)>\left(255-L_{2}\right) \\
I_{1}(i, j) \text { otherwise }
\end{array}\right.
\end{gathered}
$$

where $L_{1}=\min +\frac{a v g}{2} ; L_{2}=\max -\frac{a v g}{2} ; \min , \max$ and avg represent the minimum, maximum and average pixel intensity values of image $I_{1}(i, j)$ respectively.

$$
\begin{aligned}
& I_{4}(i, j)=\frac{1}{5} \sum_{k=1}^{5} I_{1}(i, j+k) \\
& I_{5}(i, j)=\frac{1}{5} \sum_{k=1}^{5} I_{1}(i+k, j) \\
& I_{6}(i, j)=\frac{1}{4} \sum_{i=i}^{i+1} \sum_{j=j}^{j+1} I_{1}(i, j)
\end{aligned}
$$

The fractal features $f f_{1}-f f_{6}$ were calculated from images $I_{1}-I_{6}$ and using equation (4). 


\subsection{Feature Selection}

Selecting the significant, influencing features from the set of extracted features is an important step in lung cancer detection. Extracted texture and fractal features may exhibit greater correlation or irrelevant information. All the extracted texture and fractal features may not contribute significance in classifying the lung PET/CT images as normal and abnormal. Finite size of selected features can yield an improved performance in lung cancer classification. 14 texture and 12 fractal features were extracted and analyzed. Appropriate features were selected based on their significant performance. Significant features were fed as an input to the neural network.

\subsection{Artificial Neural Network}

ANN is a promising area which has been successfully applied to various areas in medical field such as cancer detection, biochemical analysis and to detect pathological conditions. Performance of ANN depends on the selection of network parameters. Careful study was made to determine the number of input, hidden and output neurons, learning rate and momentum. Low learning rate and high momentum results in slow learning, on the contrary, no learning happens for high learning rates and low momentum. Optimal network parameters were fixed by trial and error methods.

Hidden layer size play an important role in achieving the better desired performance. Smaller number of hidden neurons makes the hidden neurons unstable, whereas larger number of hidden neurons makes the output neurons unstable ${ }^{28}$. An upper bound on the number of hidden neurons to avoid over fitting is considered as:

$$
N_{h}=\frac{N_{s}}{\alpha\left(N_{i}+N_{o}\right)}
$$

Where $N_{i}$ is number of input neurons, $N_{o}$ is number of output neurons, $N_{s}$ is number of samples in training and $\alpha$ is between 2 to 10 . Lower bound is considered as the mean value of the input and output neurons.

The set of combined image features consisting of significant features were fed as inputs to the neurons in the input layer of the network. Figure 3 shows the architecture of the proposed BPNN with optimal network parameters.

Established network was trained with the samples of known targets for various training functions. Levenberg - Marquardt back propagation is the fastest algorithm for feed forward neural networks with several hundred weights in back propagation. Network weights and bias values are updated according to Levenberg - Marquardt optimization using the Jacobian matrix of first derivatives of network errors. Hence, Levenberg - Marquardt optimization function has been utilized in the proposed

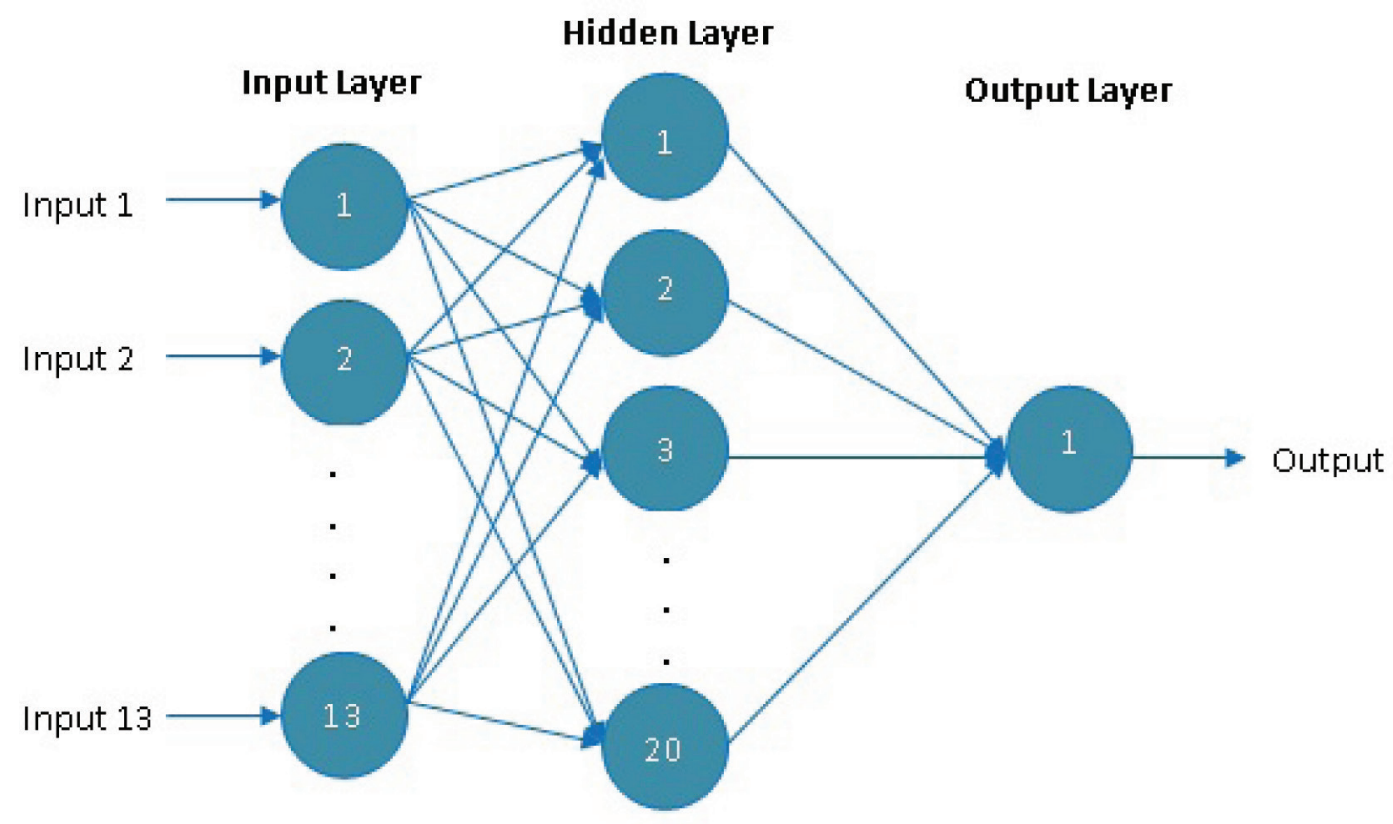

Figure 3. Architecture of the proposed back propagation neural network. 
BPNN for lung cancer classification from lung PET/CT images which involves computing around 520 weights for the extracted features. The performance of the network was computed by testing the network with new sample data and calculated the outputs. These outputs were then validated to find the testing accuracy.

\subsubsection{Performance Measures}

The performance of the classifier is evaluated by its classification accuracy. Mean Square Error (MSE), Sensitivity, specificity, accuracy and Receiver Operating Characteristic (ROC) curve are the performance measures to evaluate the performance of the classifier, as given in Equations $(12-15)$.

$$
\begin{gathered}
M S E=\frac{1}{N} \sum_{i=1}^{N} e_{i}^{2}=\frac{1}{N} \sum_{i=1}^{N}\left(\text { target }_{i}-\text { actual }_{i}\right)^{2}(12) \\
\text { Sensitivity }=\frac{T P}{T P+F N} \\
\text { Specificity }=\frac{T N}{F P+T N} \\
\text { Accuracy }=\frac{T P+T N}{\text { Totalnumberofsamples }}
\end{gathered}
$$

Where $\mathrm{TP}=$ True Positive, $\mathrm{FN}=$ False Negative, $\mathrm{FP}=$ False Positive and TN = True Negative. For a perfect classifier, the ROC curve is towards the upper-left corner (gold standard), with sensitivity and specificity of $100 \%$.

\section{Results}

DICOM images of size $256 \times 256$ were collected and the proposed method was implemented using Matlab
R2013a. Figure 4 shows few abnormal and normal lung PET/CT images from the dataset.

RGBlung PET/CT images were converted into gray scale images for reduction in complexity. Wiener filtering was applied on the gray scale images for a neighborhood of $3 \times 3$ to eliminate the additive noise present in the images. Contrast of the de-noised image was improved by using fuzzy based image processing. Gain factor ' $K$ ' in Equation (1) was fixed for appropriate enhancement. The gain factor, 0.6 was fixed after various trails with ' $K$ ' ranging between 0.1 and 0.9 . Too low and too high values of ' $K$ ' resulted in under and over enhancement respectively. Figure 5 shows the results of pre-processing.

Pre-processed images were then analyzed to extract the texture features. 14 Haralick texture features were extracted for the normal and abnormal lung PET/CT images. Fractal analysis was carried out and extracted 12 fractal features. The experimental study carried out on lung PET/CT images for fractal analysis resulted in

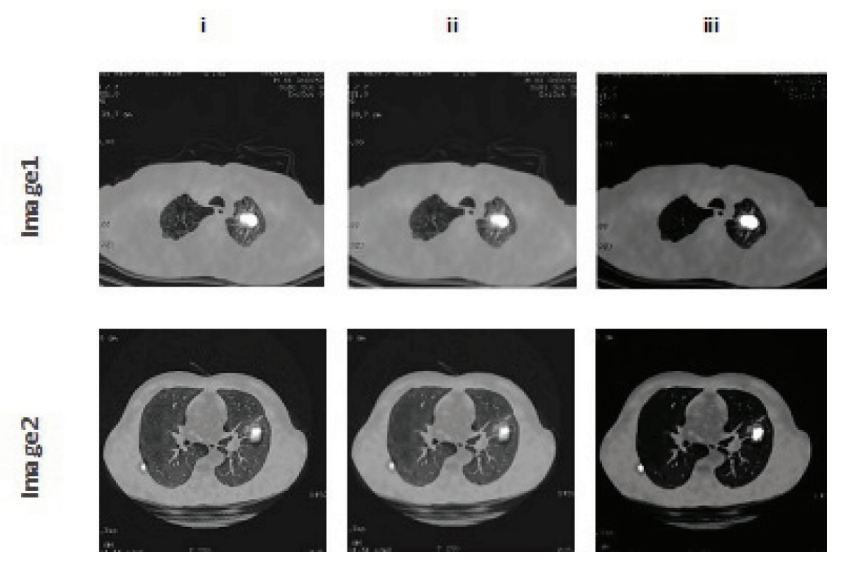

Figure 5. Preprocessed images. (a) Gray scale. (b) Wiener filtered. (c) Fuzzy enhanced. (a)

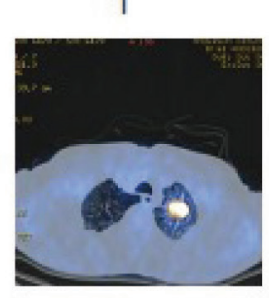

(b)

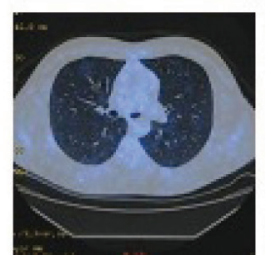

II
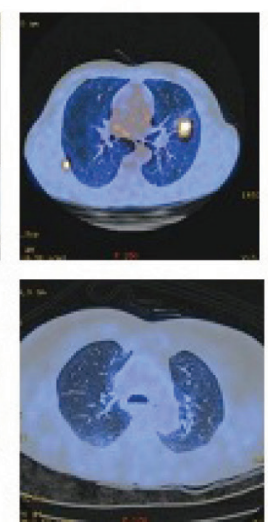

III
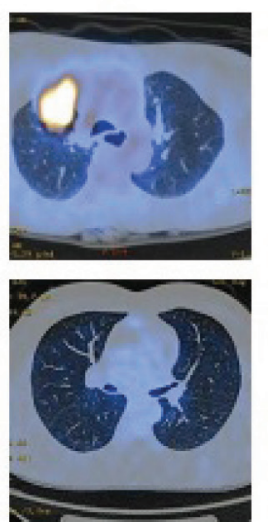

IV
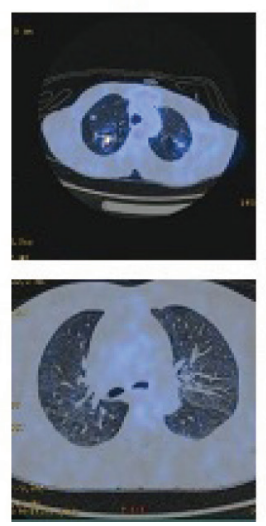

IV

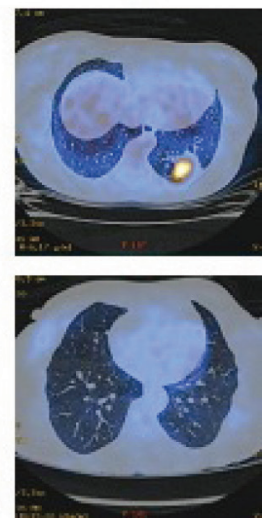

Figure 4. Sample images. (a) Abnormal. (b) Normal. 


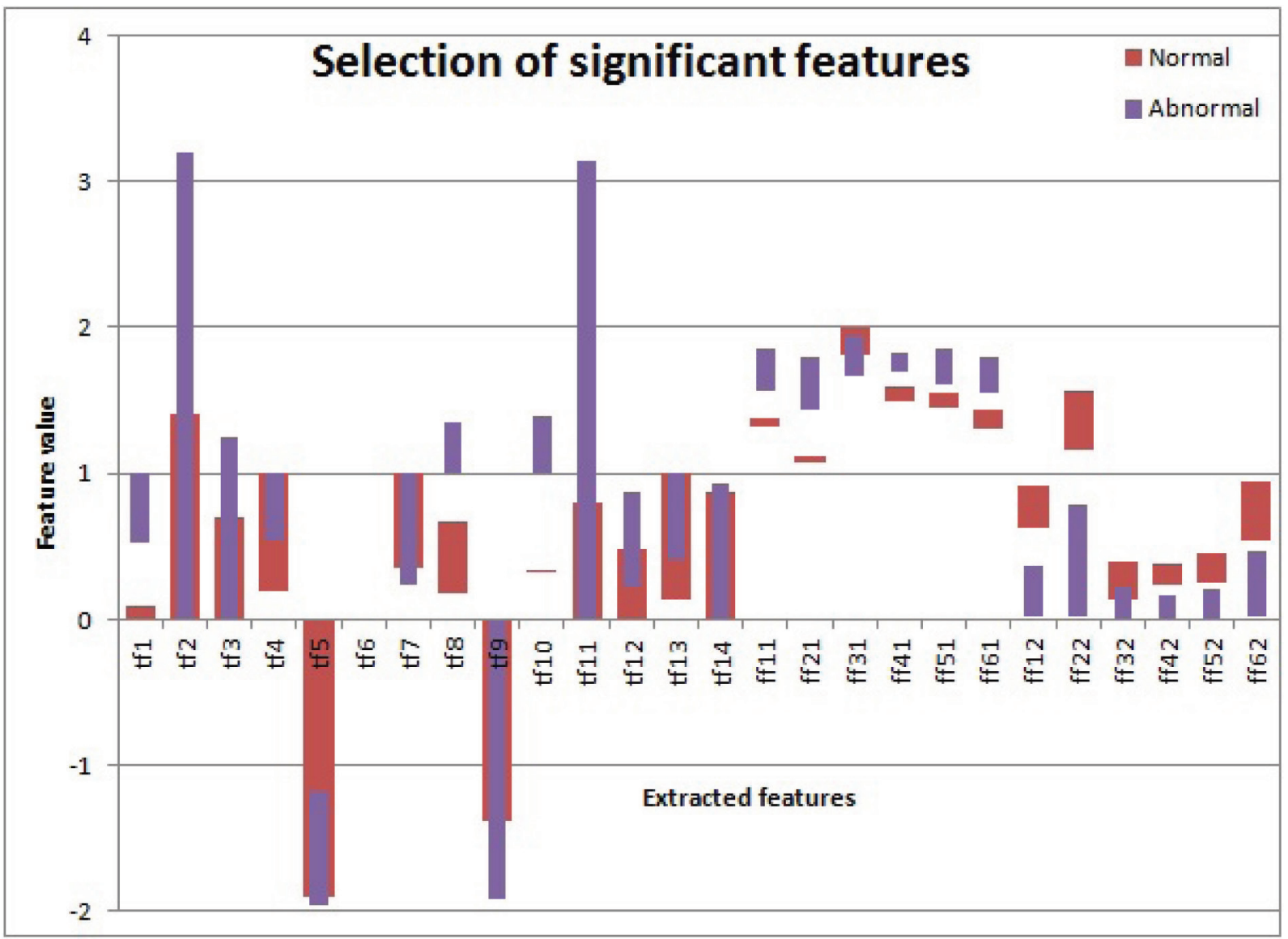

Figure 6. Plot of texture \& fractal feature values for normal and abnormal lung images.

the observation that abnormal regions of lung PET/CT images exhibit high $F D_{\text {avg }}$ and low lacunarity. The range of 14 textures $\left(t f_{1}-t f_{14}\right), 12$ fractal feature $\left(f_{11}-f_{62}\right)$ values obtained for normal and abnormal images were as shown in Figure 6.
It is observed from Figure 6 that among 14 texture features, only 3 texture features produced distinguishable values between normal and abnormal lung images. Similarly, out of 12 fractal features, 10 fractal features showed significance in classifying normal and abnormal lung images. Other texture and fractal features values
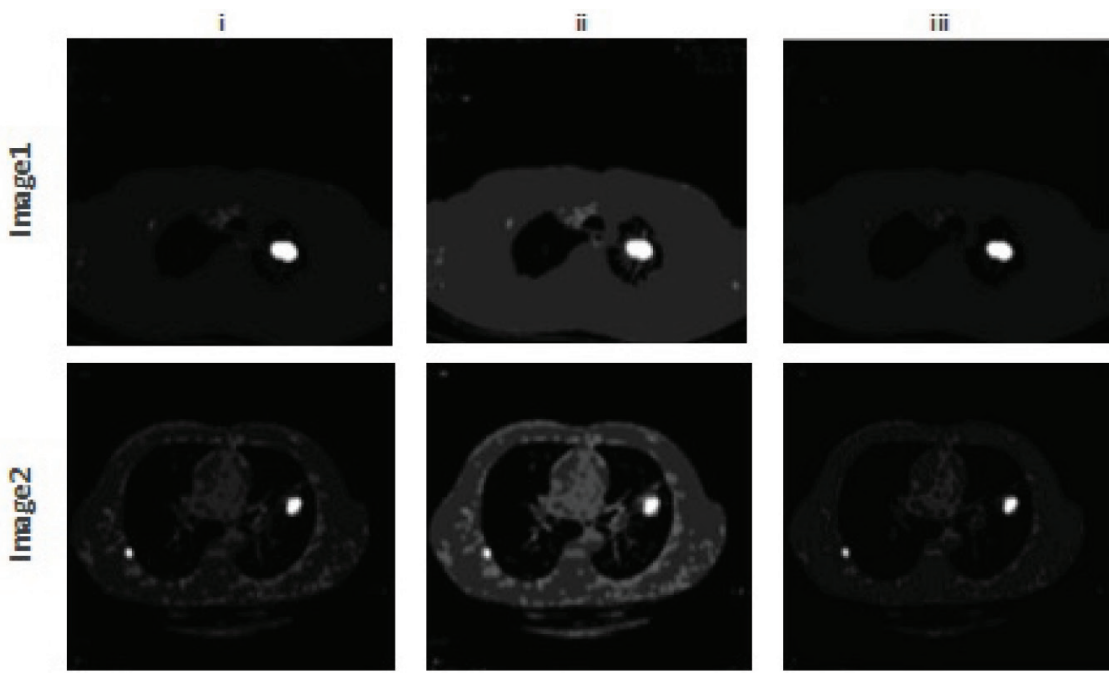

Figure 7. Significant texture features. (a) Autocorrelation. (b) Sum average. (c) Sum variance. 

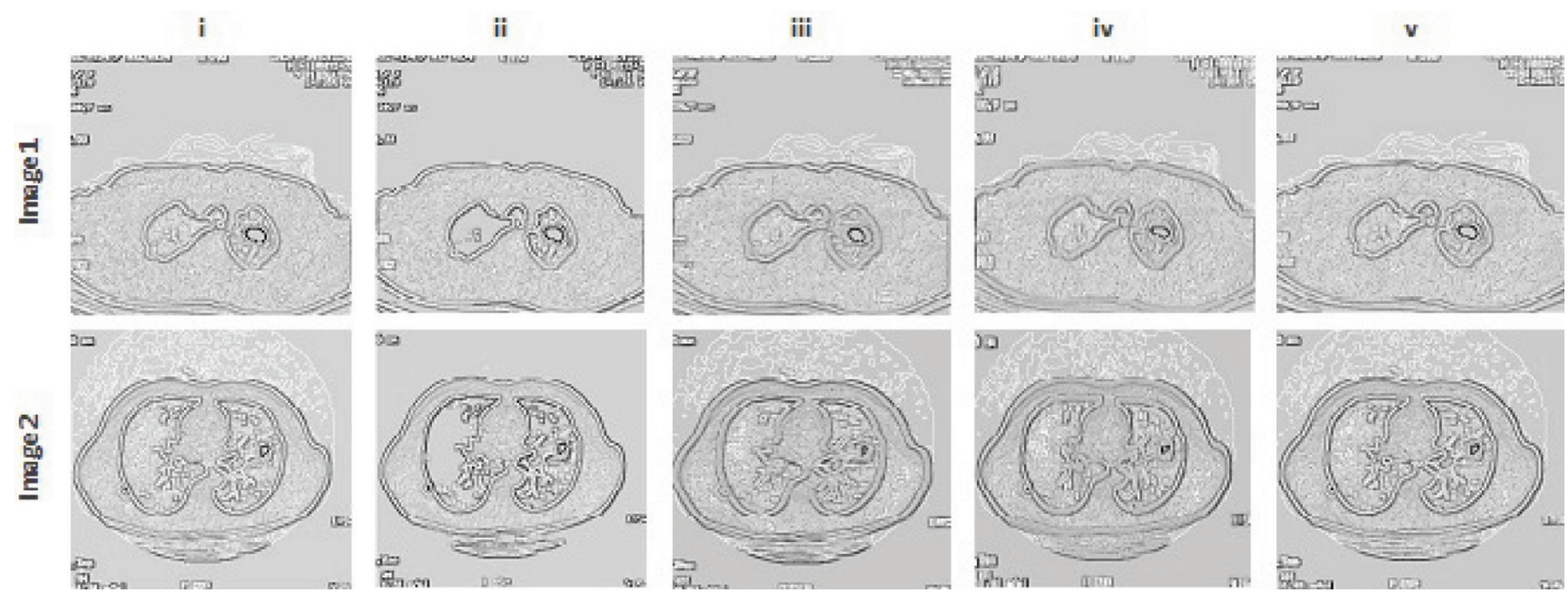

Figure 8. Significant fractal features.

were found overlapping between normal and abnormal images. Hence 3 texture features $\left(t f_{1}, t f_{8}, \mathrm{tf}{ }_{10}\right)$ and 10 fractal features $\left(f_{11}, f_{12}, f_{21}, f_{22}, f_{41}, f_{42}, f_{51}, f_{52}, f_{61}, f_{62}\right)$ were selected for classification. Figure 7 shows the significant texture features used in lung cancer detection and classification.

Figure 8 show the significant fractal features used in ANN to detect and classify the lung cancer.

Extracted texture and fractal features from samples formed the training dataset. Study on selection of network parameters was performed. Values of learning rate and momentum were tried between 0.3 and 0.9 . Keeping one value constant and changing the other parameter, performance of the classifier was studied for the performance function of MSE. Low values of learning rate and high value of momentum resulted in better learning whereas high values of learning rate and low value of momentum provided quick learning, thus saturating the network. With 1072 training samples, 13 input neurons, 1 output neuron and $\alpha=2$, the lower and upper bound of hidden neurons were found to be 7 and 38 using Equation (11) respectively. $\alpha=2$ produced better performance without over fitting. Hence, the network was trained for hidden neurons of 10,15,20,25, 30 and 35 and observed the performance. Figure 9 shows the performance graph of the training phase of network in terms of MSE and various values of hidden neurons for the combined features.

It is observed from the Figure 9 that the 20 hidden neurons produce minimum MSE for many training functions. MSE goes down as number of hidden neurons is increased from 10 in the hidden layer. But after 20 hid- den neurons MSE increases which indicates over fitting. Training functions Gradient descent with momentum, Gradient descent with adaptive learning rate \& momentum, Resilient back propagation and Scaled conjugate gradient back propagation produced best performance for 15 hidden neurons. Further testing was done with both 15 and 20 hidden neurons to evaluate the network performance. The network was tested with 80 samples of combined features. The performance results obtained for the network with the combined features for various training functions were presented in Table 2.

It is evident from the Table 2 that Levenberg Marquardt back propagation produced best classification accuracy of $98.5 \%$ (1056 samples correctly classified) and 92.5\% (74 samples correctly classified) during training and testing respectively. The performance measures such as confusion matrix and ROC curve of the classifier for the combined features during the training and testing phases is shown in Figure 10.

The training confusion matrix in Figure 10 shows that the classifier produced high sensitivity (98.3\%) and specificity (98.6\%). The testing confusion matrix shows that the classifier produced a sensitivity of $85.7 \%$ and specificity of $96.2 \%$. ROC curve for the training phase approaches the gold standard of upper left corner, thus providing the highest accuracy. The testing phase ROC curve also moves closer to the gold standard, but slightly under the training curve. The best validation performance of the classifier during training with the combined features is shown in Figure 11. 


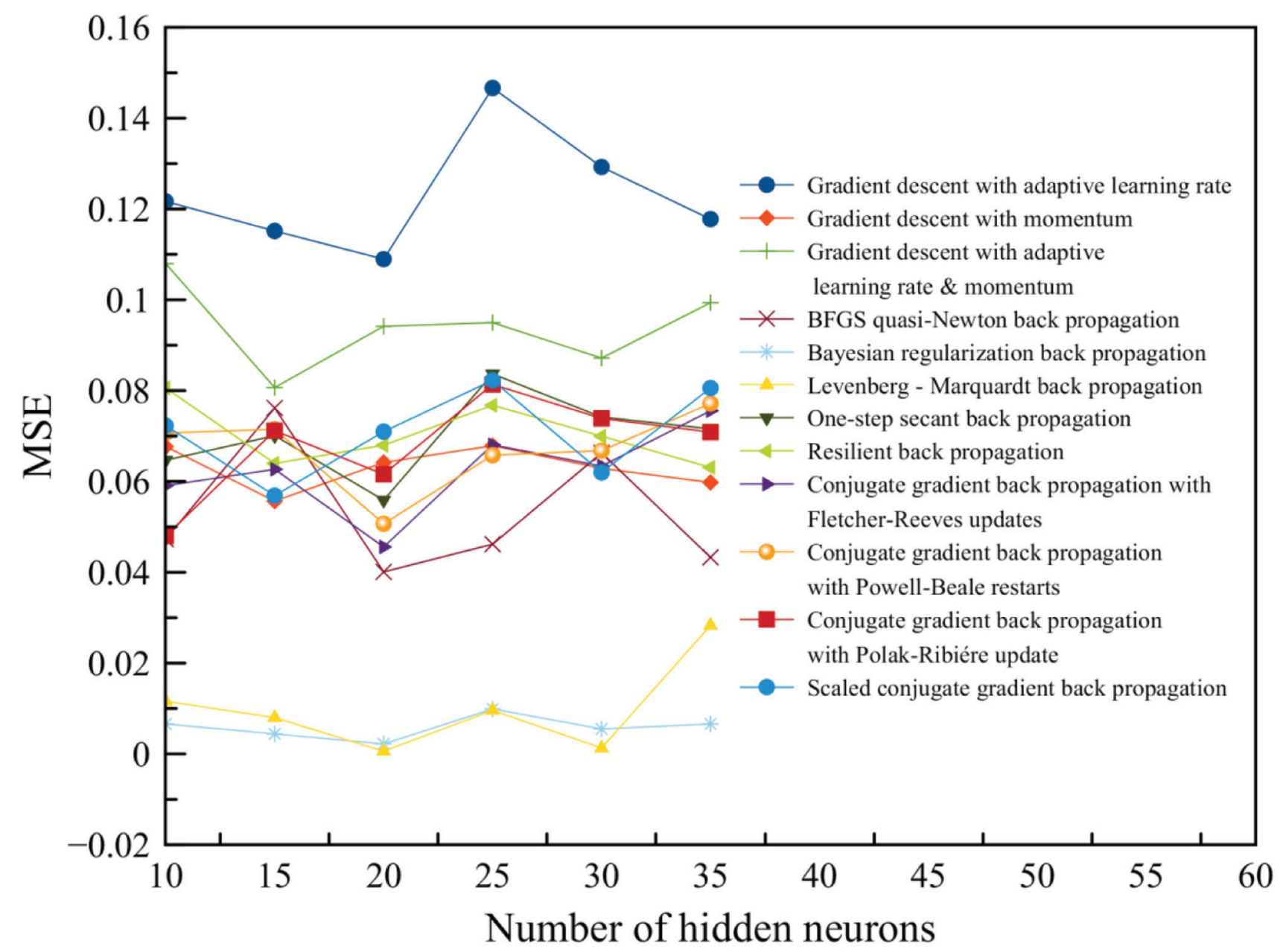

Figure 9. Performance of the network during training for various hidden neurons.

The combined features consisting of significant texture and fractal features were used in the lung cancer detection. Figure 12 shows the detected lung cancer regions and input images marked with these regions.

Table 3 shows the network parameters used in the proposed study.

\section{Discussion}

The established ANN with the optimal network parameters was trained and tested by using the 3 significant texture features, 10 fractal features and 13 combined features separately. Results obtained were tabulated and presented in Table 4.

It is clear from the performance measures in Table 4 that highest training accuracy of $98.5 \%$ was achieved by combined significant features. Highest testing accuracy was produced by fractal features and it was $95 \%$.
Combined significant features also showed a better testing accuracy of $92.5 \%$. Even though the classifier produced excellent training accuracy for the combined features, it was unable to improve the testing accuracy when significant texture features were combined with fractal features. It is noted that fractal features outperformed texture and combined features in performance measures in testing.

\section{Conclusion}

In this study, texture and fractal features have been investigated for diagnosing the lung cancer using a CAD system. The accuracy of the classifier was based upon the extracted features; training sample set size, number of hidden neurons, training functions and the network parameters. Training and testing sample dataset consists of 1072 and 80 samples respectively taken from various locations of normal and abnormal lung PET/CT images. 
Table 2. Performance results of BPNN with various training functions

\begin{tabular}{|c|c|c|c|c|c|c|c|c|}
\hline \multirow{2}{*}{ Training function } & \multirow{2}{*}{$\begin{array}{l}\text { Learning } \\
\text { rate }\end{array}$} & \multirow{2}{*}{ Momentum } & \multirow{2}{*}{$\begin{array}{l}\text { Time } \\
(\mathrm{sec})\end{array}$} & \multirow{2}{*}{ Epochs } & \multicolumn{2}{|c|}{ Training } & \multicolumn{2}{|c|}{ Testing } \\
\hline & & & & & MSE & $\begin{array}{l}\text { Accuracy } \\
(\%)\end{array}$ & MSE & $\begin{array}{l}\text { Accuracy } \\
(\%)\end{array}$ \\
\hline $\begin{array}{l}\text { Gradient descent with adaptive } \\
\text { learning rate }\end{array}$ & 0.6 & 0.3 & 21.09 & 70 & 0.1090 & 84 & 0.113 & 79.3 \\
\hline Gradient descent with momentum & 0.6 & 0.4 & 205.56 & 1000 & 0.0557 & 89.9 & 0.0744 & 87.8 \\
\hline $\begin{array}{l}\text { Gradient descent with adaptive } \\
\text { learning rate and momentum }\end{array}$ & 0.5 & 0.7 & 9.37 & 108 & 0.0828 & 86.9 & 0.0838 & 84.5 \\
\hline BFGS quasi-Newton back propagation & 0.4 & NA & 24.93 & 76 & 0.0401 & 94.8 & 0.0505 & 89.7 \\
\hline $\begin{array}{l}\text { Bayesian regularization back } \\
\text { propagation }\end{array}$ & 0.7 & 0.4 & 316.37 & 1000 & 0.0022 & 98.2 & 0.0033 & 91.8 \\
\hline $\begin{array}{l}\text { Levenberg-Marquardt back } \\
\text { propagation }^{\dagger}\end{array}$ & 0.3 & 0.9 & 22.08 & 45 & 0.0006 & 98.5 & 0.0013 & 92.5 \\
\hline One-step secant back propagation & 0.7 & 0.5 & 21.53 & 76 & 0.0559 & 90.7 & 0.0636 & 88.9 \\
\hline Resilient back propagation & 0.7 & 0.4 & 31.07 & 136 & 0.0640 & 90.3 & 0.0688 & 88.2 \\
\hline $\begin{array}{l}\text { Conjugate gradient back propagation } \\
\text { with Fletcher-Reeves updates }\end{array}$ & 0.5 & 0.8 & 9.72 & 60 & 0.0456 & 93.7 & 0.0484 & 89.0 \\
\hline $\begin{array}{l}\text { Conjugate gradient back propagation } \\
\text { with Powell-Beale restarts }\end{array}$ & 0.5 & 0.5 & 15.1 & 42 & 0.0507 & 92.5 & 0.0581 & 88.1 \\
\hline $\begin{array}{l}\text { Conjugate gradient back propagation } \\
\text { with Polak-Ribiére update }\end{array}$ & 0.5 & 0.3 & 18.51 & 66 & 0.0480 & 92.9 & 0.0501 & 88.3 \\
\hline $\begin{array}{l}\text { Scaled conjugate gradient back } \\
\text { propagation }\end{array}$ & 0.6 & 0.6 & 20.75 & 72 & 0.0569 & 88.6 & 0.0674 & 87.2 \\
\hline
\end{tabular}

Table 3. Neural network parameters in the proposed CAD system of lung cancer detection

\begin{tabular}{|l|l|}
\hline Parameters & Value \\
\hline Input Neurons & 13 (3 texture features, 10 fractal features) \\
\hline Hidden layer & 1 \\
\hline Hidden Neurons & 20 \\
\hline Output Neurons & 1 \\
\hline Training Function & Levenberg - Marquardt back propagation \\
\hline Learning Rate & 0.3 \\
\hline Momentum & 0.9 \\
\hline Maximum number of epochs & 1000 \\
\hline
\end{tabular}

Table 4. Performance measures of the classifier with texture, fractal and combined features

\begin{tabular}{|l|l|l|l|l|l|l|}
\hline & \multicolumn{2}{|l|}{ Texture Features } & \multicolumn{2}{l|}{ Fractal Features } & \multicolumn{2}{l|}{ Combined significant features } \\
\cline { 2 - 7 } & Training & Testing & Training & Testing & Training & Testing \\
\hline Sensitivity (\%) & 94.2 & 82.1 & 95.5 & 85.7 & 98.3 & 85.7 \\
\hline Specificity (\%) & 90.8 & 96.2 & 100 & 100 & 98.6 & 96.2 \\
\hline Accuracy (\%) & 92.4 & 91.3 & 98.1 & 95 & 98.5 & 92.5 \\
\hline
\end{tabular}



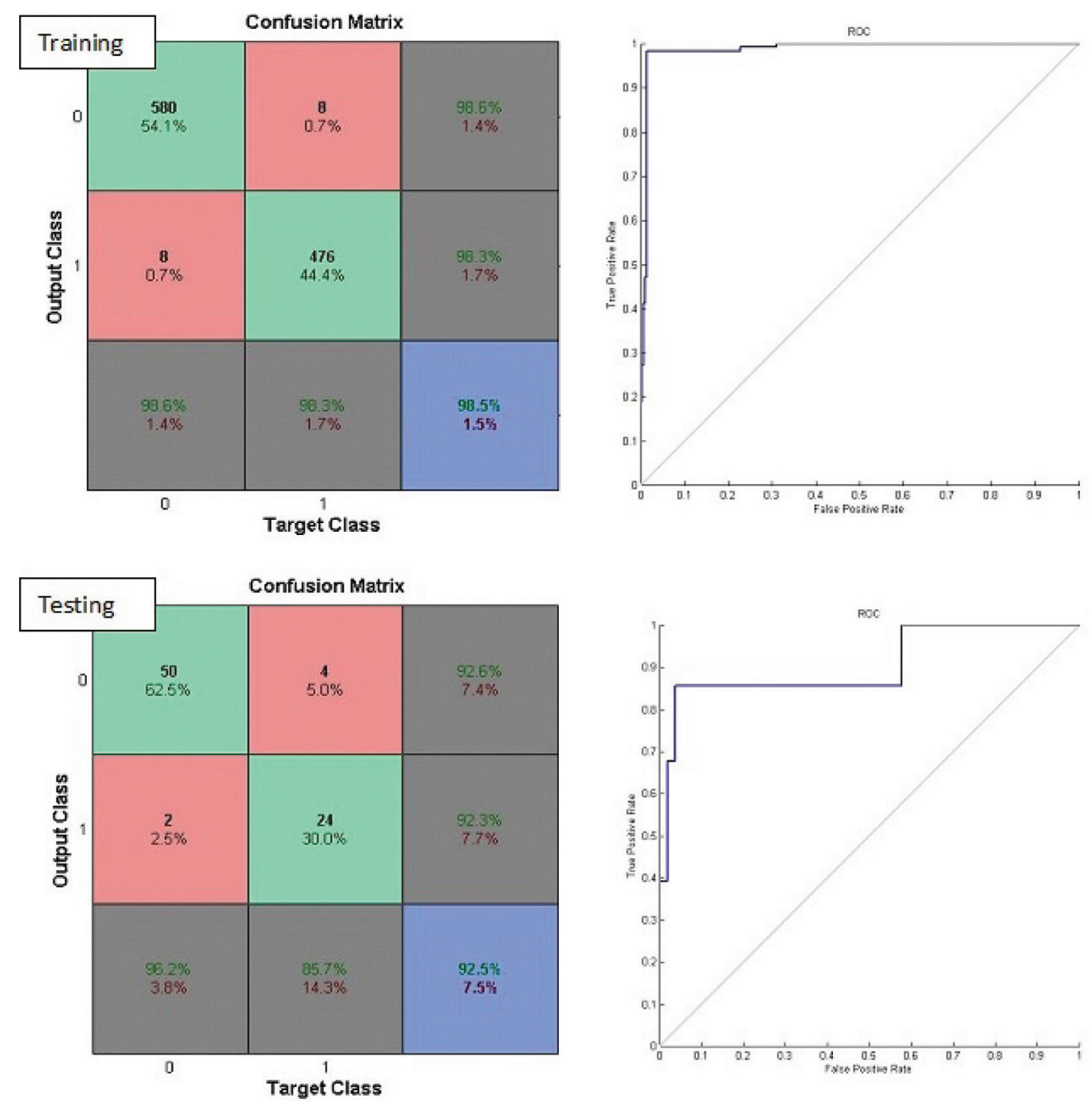

Figure 10. Confusion matrix and ROC curve of the classifier with combined features.

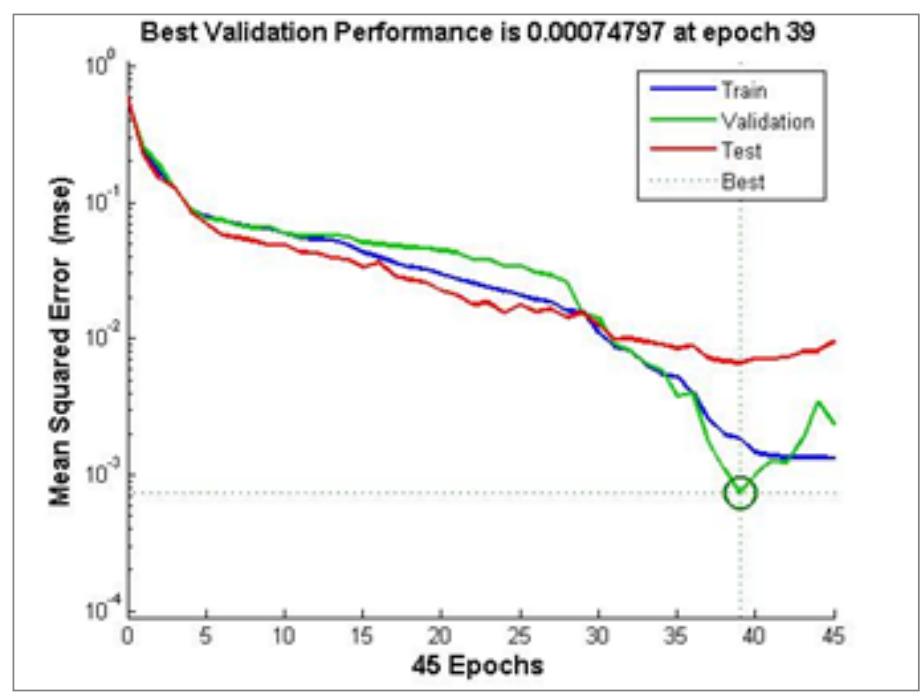

Figure 11. Best validation performance of the classifier

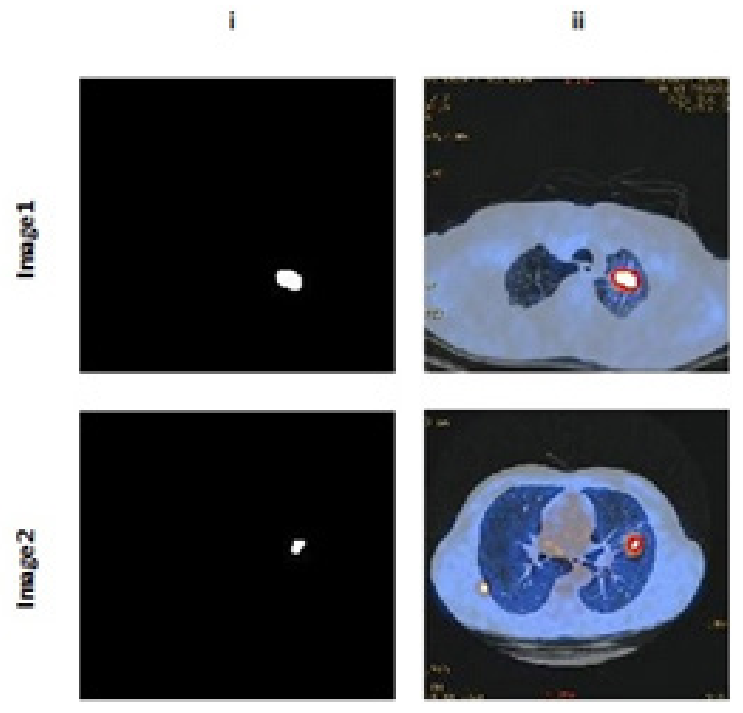

Figure 12. Lung cancer detected and input images marked with the detected cancer regions 
Selected combined feature set included 3 texture and 10 fractal features. Proposed method yielded better classification accuracy for training and testing with Levenberg - Marquardt back propagation for a learning rate of 0.3 , momentum of 0.9 and 20 hidden neurons. The testing accuracy achieved with the proposed method for fractal features was $95 \%$ and combined features resulted in a testing accuracy of $92.5 \%$. The accuracy can be compared with other supervised classifiers and larger datasets.

\section{Acknowledgement}

The authors thank Anderson Diagnostics \& Labs, Chennai for the dataset, valuable inputs and validation of the output. Authors also thank the management of Hindustan Institute of Technology \& Science for their consistent support and encouragement to accomplish the work.

\section{References}

1. Ali I, Wani WA, Saleem K. Cancer scenario in India with future perspectives. Cancer Therapy. 2011 Jan; 8:56-70.

2. Noronha V, Pinninti R, Patil VM, Joshi A, Prabhash K. Lung cancer in the Indian subcontinent. South Asian Journal of Cancer. 2016 Jul; 5(3):95-103. Crossref. PMid:27606290 PMCid:PMC4991146

3. Behera D, Balamugesh T.Lung cancer in India. Indian Journal ofChestDiseasesandAlliedSciences.2004Oct;46(4):269-82. PMid:15515828

4. Mohan A, Latifi AN, Guleria R. Increasing incidence of adenocarcinoma lung in India: Following the global trend? Indian Journal of Cancer. 2016 Jan; 53(1):92-5. Crossref. PMid:27146752

5. Parikh PM, Ranade AA, Govind B, Ghadyalpatil N, Singh R, Bharath R, Bhattacharyya GS, Koyande S, Singhal M, Vora A, Verma A. Lung cancer in India: Current status and promising strategies. South Asian Journal of Cancer. 2016 Jul; 5(3):93-5. Crossref. PMid:27606289 PMCid:PMC4991145

6. Tsukamoto E, Ochi S. PET/CT today: System and its impact on cancer diagnosis. Annals of Nuclear Medicine. 2006 May; 20(4):255-67. Crossref. PMid:16856569

7. Chao F, Zhang H. PET/CT in the staging of the non-smallcell lung cancer. BioMed Research International. 2012 Mar; 2012:1-8.

8. Castellino RA. Computer Aided Detection (CAD): An overview. Cancer Imaging. 2005 Aug; 5(1):17-9. Crossref. PMid:16154813 PMCid:PMC1665219

9. Firmino M, Morais AH, Mendoça RM, Dantas MR, Hekis $\mathrm{HR}$, Valentim R. Computer-aided detection system for lung cancer in computed tomography scans: Review and future prospects. Biomedical Engineering Online. 2014 Apr; 13(1):41. Crossref. PMid:24713067 PMCid:PMC3995505

10. Kalyan K, Jakhia B, Lele RD, Joshi M, Chowdhary A. Artificial neural network application in the diagnosis of disease conditions with liver ultrasound images. Advances in Bioinformatics. 2014 Sep; 2014:14.

11. Pourahmad S, Azad M, Paydar S. Diagnosis of malignancy in thyroid tumors by multi-layer perception neural networks with different batch learning algorithms. Global Journal of Health Science. 2015 Nov; 7(6):46-54. Crossref. PMid:26153161 PMCid:PMC4803901

12. Soumya MK, Sneha K, Arunvinodh C. Cervical Cancer Detection and Classification using Texture Analysis. Biomedical and Pharmacology Journal. 2016 Jun; 9(2):66371. Crossref.

13. Sharma B. Comparison of neural network training functions for hematoma classification in brain CT images. IOSR Journals (IOSR Journal of Computer Engineering). 2014 Jan; 1(16):31-5. Crossref.

14. Irianto BG, Mak'ruf MR, Titisari D. Identification of lung cancer using a back propagation neural network. Indonesian Journal of Electrical Engineering and Computer Science. 2015 Oct; 16(1):91-7.

15. Hussain MA, Ansari TM, Gawas PS, Chowdhury NN. Lung cancer detection using artificial neural network \& fuzzy clustering. International Journal of Advanced Research in Computer and Communication Engineering. 2015 Mar; 4(3):360-3. Crossref.

16. Kuruvilla J, Gunavathi K. Lung cancer classification using neural networks for CT images. Computer Methods and Programs in Biomedicine. 2014 Jan; 113(1):202-9. Crossref. PMid:24199657

17. Almas P, Bariu KS. Detection and classification of lung cancer using artificial neural network. International Journal on Advanced Computer Engineering and Communication Technology. 2012; 1(1):62-7.

18. Kohad R, Ahire V. Application of machine learning techniques for the diagnosis of lung cancer with ANT colony optimization. International Journal of Computer Applications. 2015 Jan; 113(18):34-41. Crossref.

19. Taher F, Werghi N, Al-Ahmad H, Sammouda R. Lung cancer detection by using artificial neural network and fuzzy clustering methods. American Journal of Biomedical Engineering. 2012; 2(3):136-42. Crossref.

20. Penedo MG, Carreira MJ, Mosquera A, Cabello D. Computer-aided diagnosis: A neural-network-based approach to lung nodule detection. IEEE Transactions on Medical Imaging. 1998 Dec; 17(6):872-80. Crossref. PMid:10048844 
21. Al-Kadi OS, Watson D. Texture analysis of aggressive and nonaggressive lung tumor CE CT images. IEEE Transactions on Biomedical Engineering. 2008 Jul; 55(7):1822-30 Crossref. PMid:18595800

22. Blodgett TM, Mehta AS, Mehta AS, Laymon CM, Carney J, Townsend DW. PET/CT artifacts. Clinical Imaging. 2011 Feb; 35(1):49-63. Crossref. PMid:21237418 PMCid:PMC4277262

23. Magdy E, Zayed N, Fakhr M. Automatic classification of normal and cancer lung CT images using multiscale AM-FM features. Journal of Biomedical Imaging. 2015 Jan; 2015:7.

24. Haralick RM, Shanmugam K. Textural features for image classification. IEEE Transactions on Systems, Man and Cybernetics. 1973 Nov; 3(6):610-21. Crossref.
25. Punithavathy K, Ramya MM, Poobal S. Analysis of statistical texture features for automatic lung cancer detection in PET/ CT images. IEEE Proceedings of International Conference on Robotics Automation Control and Embedded Systems (RACE); 2015 Feb. p. 1-5.

26. Chaudhuri BB, Sarkar N. Texture segmentation using fractal dimension. IEEE Transactions on Pattern Analysis and Machine Intelligence. 1995 Jan; 17(1):72-7. Crossref.

27. Sankar D, Thomas T. Fractal features based on differential box counting method for the categorization of digital mammograms. Journal of Computer Information Systems and Industrial Management Applications. 2010; 2:11-9.

28. Karsoliya S. Approximating number of hidden layer neurons in multiple hidden layer BPNN architecture. International Journal of Engineering Trends and Technology. 2012 Dec; 3(6):714-7. 